\title{
The Internet of Molecular Things Based on FRET
}

\author{
Murat Kuscu, Student Member, IEEE and Ozgur B. Akan, Senior Member, IEEE
}

\begin{abstract}
Molecular devices, which consist of single or a few molecules, are envisioned to perform advanced tasks such as molecular information processing and collaborative sensing/actuating if they are operated in a cooperative manner. To connect these nanoscopic primitive devices with each other and with macroscale networks, and thus, to realize the internet of molecular devices, requires fundamentally different and novel approaches, other than the molecular or electromagnetic nanocommunications. Recently, we proposed and studied the use of Förster Resonance Energy Transfer (FRET), which is a shortrange nonradiative energy transfer process between fluorophores, as a high-rate and reliable wireless communication mechanism to connect fluorophore-based photoactive molecular devices. In this paper, we provide an in-depth architectural view of this new communication paradigm with a focus on its peculiarities, fundamental principles and design requirements by comprehensively surveying the theoretical and experimental positions and ideas. We give an overview of networking opportunities offered by the intrinsic capabilities of fluorophores under the novel concept of Internet of Molecular Things. We present some prospective applications, theoretical modeling approaches and experimental opportunities, and finally discuss the implementation challenges.
\end{abstract}

Index Terms-FRET, fluorophores, molecular devices, nanonetworks, internet of things

\section{INTRODUCTION}

$\mathbf{F}$ LUOROPHORES are a special class of molecules, including fluorescence proteins, organic dyes, quantum dots (QDs), which can be optically excited and fluoresce at visible wavelengths, thus, serve as interface between nanoscale and macroscale worlds [1]. Their use in biological imaging started the era of fluorescence microscopy which then evolved to the fluorescence nanoscopy with nanometer resolution, the development of which was awarded the 2014 Nobel Prize in Chemistry [2]. Besides their extensive use as molecular beacons, they have been also functionalized to serve as binary logical switches which are controllable by several kinds of stimuli [3], [4], and memory units [5], as well as actuators [6]. Connecting these molecular devices with each other and with macroscale networks in a controllable manner is envisaged to enable advanced applications including molecular all-optical information processing and small scale sensor-actor nanonetworks performing cooperative theranostic tasks which are far beyond the capabilities of individual devices.

Prominent approaches to nanoscale communications, such as the use of molecules or THz-band electromagnetic waves

The authors are with the Next-generation and Wireless Communications Laboratory (NWCL), Department of Electrical and Electronics Engineering, Koc University, Istanbul, 34450, Turkey (e-mail: \{mkuscu, akan $\} @$ ku.edu.tr).

This work was supported in part by the European Research Council (ERC) under grant ERC-2013-CoG \#616922, by the Turkish Scientific and Technical Research Council under grant \#109E257, by the Turkish National Academy of Sciences Distinguished Young Scientist Award Program (TUBA-GEBIP), and by IBM through IBM Faculty Award. as information carriers [7], [8], do not provide a feasible solution for implementing networks of molecular scale devices, because they include overly complex communication mechanisms which obviously cannot be handled by these primitive devices consisting of single or a few molecules with dimensions lower than $100 \mathrm{~nm}$. In the quest to find an alternative approach, we proposed to exploit FRET as a nanoscale wireless communication mechanism between fluorophore-based devices [9].

FRET is the nonradiative exchange of electronic states between an excited-state fluorophore serving as donor and a ground-state fluorophore serving as acceptor when they are in close proximity, i.e., $0-10 \mathrm{~nm}$, of each other [10]. As a result of FRET, the donor returns to its ground-state whereas the acceptor becomes excited. In addition to the proximity condition, FRET also requires for the donor-acceptor pair to be spectrally similar to enable resonance. The probability and rate of transfer strongly depend on the degree of fulfillment of these criteria, and also on the intrinsic properties of participating fluorophores. The phenomenon has a well-established theory which is validated over years of prevalent use in biotechnology applications as spectroscopic rulers.

We have envisioned FRET as a communication mechanism between fluorophore-based molecular devices through encoding information into the electronic states of molecules. Toward realizing this vision, we have initially focused on developing theoretical models for possible network configurations. In [9], we provided the physical channel model between a single pair of fluorophores, and derived its information theoretical capacity. A robust encoding scheme using multiple molecular excitons as information carriers was introduced and analyzed for point-to-point and point-to-multipoint channels in [11]. In the same work, we also investigated potential mechanisms of external control on the communication pathways. A multihop relay topology extending the range of information transfer to a few hundred nanometers was provided in [12]. Using functionalized fluorophores as network nodes, we proposed a mobile ad hoc sensor-actor nanonetwork in [13], and examined its coverage and throughput performance in [14]. Finally, to prove the feasibility of FRET-based nanocommunications, in [15], we demonstrated the first practical data transfer between organic dye fluorophores at the transmitting rates of 50, 150 and $250 \mathrm{kbps}$ with very low error rates. These studies underlined the potential and practicality of FRET as a highrate and reliable nanocommunication means among molecular scale devices.

In this paper, our objective is to provide an in-depth coverage of the basic principles of FRET-based communications. We first present a conceptual view of the envisioned architecture, and elaborate on the design options for each phase of communication. We examine the networking opportunities, 


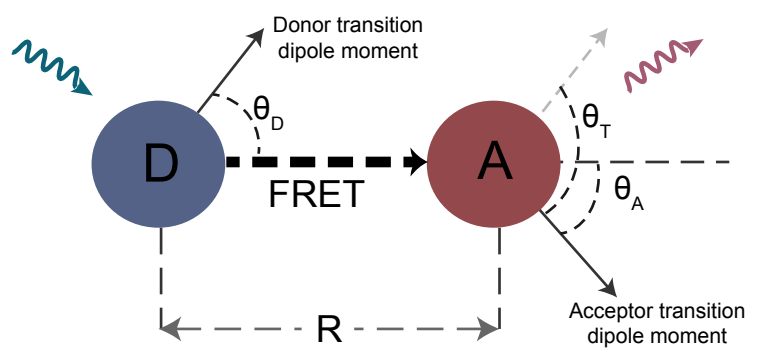

Fig. 1. FRET between an excited-state donor fluorophore and a ground-state acceptor fluorophore. $\theta_{T}$ denotes the angle between the emission transition dipole of the donor $\mathbf{D}$ and the absorption transition dipole of the acceptor $\mathbf{A}$, $\theta_{D}$ and $\theta_{A}$ denote the angles between these dipoles and the vector joining the donor and acceptor. $R$ is the intermolecular distance.

offered by fluorophore and FRET technologies, by reviewing the related theoretical and empirical positions and ideas. We then investigate the feasibility of interfacing FRET-based nanonetworks with macroscale networks to realize the internet of molecular things, and give an overview of prospective applications. We present the theoretical modeling approaches together with the simulation algorithms developed for different network configurations. We lastly discuss the experimental opportunities and implementation challenges.

The remainder of the paper is organized as follows. In Section II, we give an overview of FRET phenomenon together with the main properties of fluorophores. In Section III, we provide the conceptual architecture of FRET-based nanocommunications and discuss the networking opportunities. In Section IV, we present the prospective applications. Theoretical modeling approaches are given in Section V. Experimental opportunities and implementation challenges are discussed in Section VI. Finally, the concluding remarks are given in Section VII.

\section{OVERVIEW OF FLUOROPHORES AND FRET}

Fluorophores are chemical compounds that can emit a photon upon the excitation by an optical stimulation. The emitted photon is generally of lower energy than the excitation photon due to the energy loss in vibrational energy states. Time between the excitation and relaxation of a fluorophore is exponentially random, mean of which is termed as excitedstate lifetime, and is on the order of nanoseconds for common fluorophores [1]. Organic dyes, fluorescent proteins, semiconductor QDs are the most frequently used fluorophores as molecular probes in fluorescence spectroscopy.

Releasing a photon, i.e., fluorescing, is not the only relaxation pathway for an excited fluorophore. The radiative relaxation could be hindered by a competing quenching mechanism. For example, collision or reaction of the excited fluorophore with another molecule may result in the dissipation of excited-state energy as heat leading to the quenching of the fluorophore. However, probability of molecular collision or any pairwise reaction during the lifetime of excited-state is very low even in bulk solutions, therefore, these relaxation pathways are generally neglected [1]. Another fundamental excited-state process is FRET, which defines the nonradiative transfer of excited-state energy to a nearby ground-state fluorophore. As a result of FRET, while the emission of initially excited fluorophore, serving as energy donor, is being quenched; another flurophore, serving as energy acceptor, is excited leading to sensitized emission with a red-shifted wavelength as demonstrated in Fig. 1. The phenomenon is resultant from the long-range dipole-dipole Coulomb interactions of excited- and ground-state fluorophores.

FRET is feasible only when the following conditions are met: $i)$ the donor fluorophore should be in close proximity $(0-10 \mathrm{~nm})$ with at least one ground-state acceptor; ii) the absorption spectrum of the acceptor should have a considerable overlap with the emission spectrum of the donor; and iii) the absorption transition dipole moment of the acceptor should not be orthogonal to the emission transition dipole of the donor. When these conditions are satisfied, FRET becomes a possible relaxation pathway for the excited-state donor and competes with the natural fluorescence process. Through this competition, FRET decreases the excited-state lifetime and the fluorescence probability of the fluorophore. The probability of FRET depends on the degree of fulfillment of these conditions together with the intrinsic properties of the donor and acceptor.

To abstract the excited-state processes, it is beneficial to consider the excited-state of fluorophores as particle-like molecular excitons [16], which can be generated on fluorophores as a result of excitation or sensitization, converted to a photon via fluorescence, or transferred among fluorophores via FRET. In this way, FRET becomes a process defining the hopping of excitons on a lattice consisting of fluorophore nodes. The rules governing the exciton dynamics can be summarized as follows:

- A fluorophore node can be occupied by at most one exciton. Thus, to accept an exciton transfer from another fluorophore, it should lose its exciton, if any, and return to the ground-state.

- An exciton has a short and random lifetime, i.e., sojourn time, on a fluorophore. When there are multiple relaxation pathways for the fluorophore leading to multiple competing processes, the lifetime further decreases.

- Excitons do not have transferable properties, since they only represent the state of being excited on the occupied fluorophore.

- Once converted into a photon or transferred to another fluorophore, an exciton is removed from the initially occupied fluorophore without any trace.

- Excitons cannot be copied or stored on a fluorophore.

\section{FRET-BASED NANOSCALE COMMUNiCATIONS}

Being the short-range but high-rate transfer of excitons between fluorophores, FRET could be exploited as a nanoscale wireless communication method to connect individual molecular devices by encoding information into excitons. This section provides a conceptual architecture for this communication method having very interesting peculiarities due to the quantized nature of excitons, and elaborates on the networking opportunities. 


\section{A. Architecture}

A communication process can be viewed as comprising basically five consecutive phases: encoding, transmitting, propagation, receiving, and decoding. FRET-based nanoscale communications, due to the lack of an actual propagation channel and the instantaneous nature of exciton transfer, consists of encoding and decoding phases, and an additional transfer phase combining the transmitting, propagation and receiving processes. We explain each phase in detail as follows:

1) Encoding Phase: In the encoding phase, information is translated into a representation by excitons which can be transmitted through FRET channel. This requires for the controllable generation of excitons on the transmitter node. The excitation source could be an external optical device such as a laser which can emit photons with wavelengths matching to the absorption spectrum of the transmitter fluorophore. The device could be the direct source of information if it is regulated by an additional computing device or connected to a macroscale network, or it could be used only for the purpose of continuously powering the transmitter to generate excitons for encoding information related to a nanoscale phenomenon such as a sensing event. If the transmitter is a bio/chemoluminescent molecule, then, a chemical reaction resulting in excitation of the transmitter could be both power and information source [17].

Depending on the excitation source and method, a proper modulation scheme should be applied. Given the fact that a single exciton as information carrier represents only the state of the occupied fluorophore and does not possess transferable properties such as amplitude or frequency, the number of feasible modulation methods are very limited. The only appropriate amplitude modulation scheme is On-Off Keying (OOK), which can be implemented by encoding logical bit- 1 and bit- 0 into the presence and absence of excitons for a predefined time interval [9]. If an optical laser is employed as the power or information source, depending on the length of optical pulses, single excitons or sequentially generated multiple excitons can represent bit-1 [12]. If the excitation is realized through an irreversible chemical reaction, then encoding into single exciton is the only way of representing information. For the optical excitation case, pulse modulation methods such as pulse-width modulation, pulse-duration modulation and pulse-frequency modulation are also feasible for FRET-based communications [18].

2) Transfer Phase: Different from the electromagnetic and molecular communications, FRET-based communications, due to the nonradiative nature of FRET, does not include a propagation phase, in which information carriers propagate from a transmitter to a receiver [1]. Moreover, transmission of information-carrying signals by a transmitter and their reception by a receiver are simultaneous processes, since excitons are instantly transferred. Therefore, transmitting, propagating and receiving phases can be jointly viewed as a single transfer phase.

Transfer phase requires for the FRET criteria to be satisfied such that there should be at least one suitable receiver in the transfer range of the transmitter. If the criteria are met, an information-carrying exciton generated on the transmitter is transferred to the receiver node at a random time. The time interval between the generation and transfer of a single exciton cannot be controlled by the transmitter, and solely depends on the extent and number of the present relaxation pathways. For example, as the receiver node gets closer to the transmitter, exciton generation and transfer rates are increased along with the decreasing sojourn time of excitons on the transmitter. Since fluorescence is an intrinsic relaxation pathway, there is always a chance that a generated exciton is recombined into a photon before transferred. This implies the loss of information if it is encoded by a single exciton. From this aspect, multiexciton scheme that uses longer excitation pulses to encode information into sequentially generated multiple excitons is more robust compared to single-exciton schemes, although it reduces the communication rate [11].

It is to be noted here that there is an exceptional case for the propagation process, which is the usage of a plasmonic waveguide as the intermediary of exciton transfer [19], [20]. In this configuration, a pair of transmitter and receiver is immobilized on the surface of a plasmonic nanostructure, e.g., two-dimensional doped graphene [21]. Internodal distance between the transmitter and receiver can be set larger than the FRET range. An exciton generated on the transmitter is first transferred to the propagation channel by resonantly exciting a surface plasmon. The excited plasmon propagates through the waveguide and carries the information radiatively. If it succeeds in arriving at the receiver location, the receiver can be resonantly excited by the surface plasmon. In this way, the information can be transferred much longer distances with the collaboration of exciton transfer and surface plasmon propagation.

3) Decoding Phase: In the decoding phase, received excitons are transduced into another signal form, which can be processed or used for realizing a proper action in the context of a nanonetwork application. The receiving node has no control over the decoding phase, and realizes its predefined functionality in an autonomous manner. As it receives excitons from the transmitter, it can fluoresce to optically transmit the information to an external observer. In addition, transduction of received excitons into electrical signals at the nanoscale can also be feasible with the use of semiconductor nanowire photodiodes [22]. Moreover, if the receiver node is a photosensitizer fluorophore, excitons could be used to sensitize singlet oxygens in photodynamic therapy of cancer [6], which will be further discussed in Section IV.

\section{B. Networking Opportunities}

Most of the envisioned nanotechnology applications require more than two molecular devices to communicate with each other, thus, to form nanonetworks that can accomplish advanced tasks [23]. Due to the inability of individual fluorophore nodes to process signals and self-control, FRET-based nanonetworks are envisaged to be passive-networks with predefined autonomous or externally-controllable functionalities [13]. In this section, we give an overview of the feasible networking opportunities offered by the intrinsic capabilities of fluorophores and FRET. 
(a)

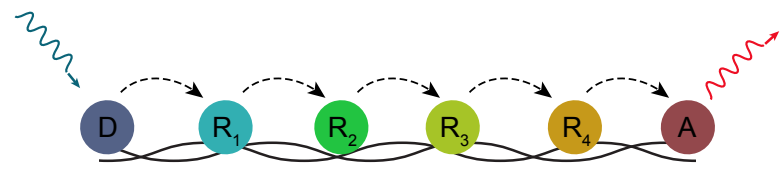

(b)

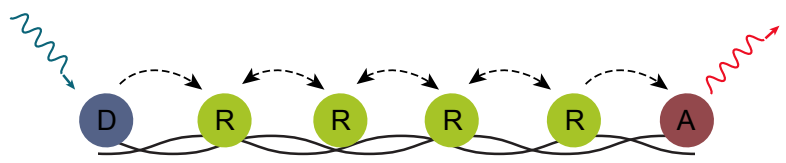

(c)

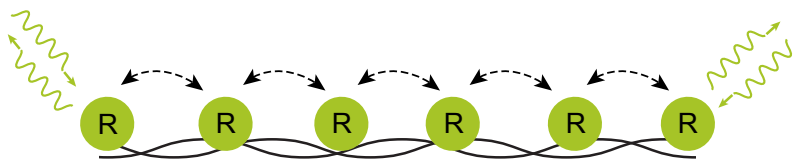

(d)

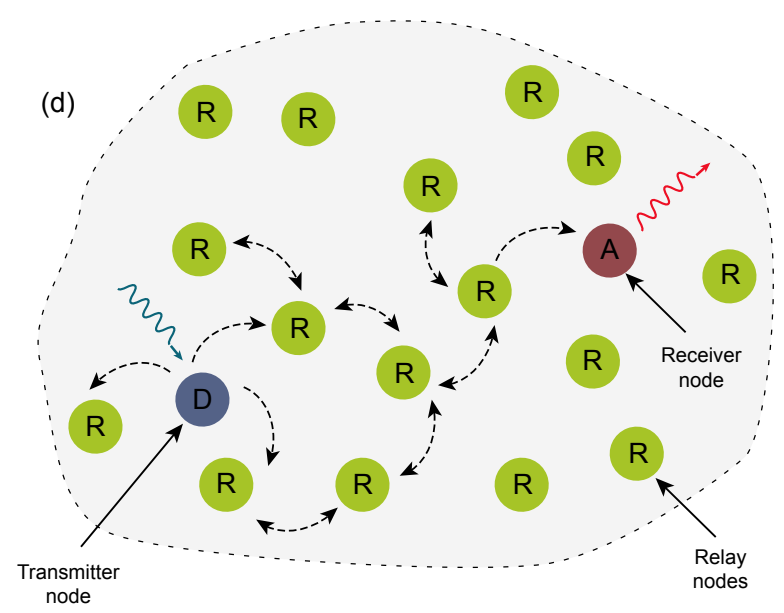

Fig. 2. Multihop FRET-based communication schemes: (a) 1-d unidirectional photonic wire on DNA scaffold consisting of spectrally different fluorophores; (b) 1-d unidirectional photonic wire consisting of identical relay fluorophores between a transmitter and a receiver node; (c) 1-d bidirectional photonic wire consisting of identical fluorophores acting as transmitter, relay, and receiver nodes; (d) 3-d multihop link consisting of randomly deployed relay nodes between a transmitter-receiver node pair.

1) Multihop Communication: Limited range of FRETbased communications can be extended via multihop FRET, which is the sequential transfer of an exciton through cascaded FRET channels [24]. Cascade of channels can be realized deploying a relay node between the transmitter and receiver nodes, with an absorption spectrum overlapping the emission spectrum of the transmitter, and an emission spectrum overlapping the absorption spectrum of the receiver [9]. In this way, an information carrying exciton generated on the transmitter is first transferred to the relay node. Then the relay node, without any amplification mechanism, autonomously transfers the exciton to the receiver node if the FRET criteria are satisfied for both connections. Likewise, we can employ multiple relay nodes to further extend the communication range by increasing the number of cascaded channels, all of which satisfy the FRET criteria [12]. If each of the utilized relay fluorophores is of different type, their emission and absorption spectra should be red-shifted as their location approaches to the receiver node to ensure the directionality of the information flow as shown in Fig. 2(a). Relays can also be identical if their emission and absorption spectra do a significant overlap (see Fig. 2(b)). In this case, the transfer between identical fluorophores is termed homoFRET [1]. If the overall network nodes including the transmitter and receiver are all identical, homoFRET among them can enable communication in both direction in a halfduplex manner (see Fig. 2(c)).

Depending on the application and the required communication range, relays can be deployed in numerous configurations. They can be immobilized on a one dimensional structure to obtain a one dimensional photonic wire. DNA and zeolite L are the examples of one dimensional host molecules allowing the deployment of organic dyes on prescribed locations [24], [25]. Relays can also be mobile randomly moving in a three dimensional fluidic environment as demonstrated in Fig. 2(d). The excitons randomly hop along a random lattice comprised of mobile relays, and communication is realized in an ad hoc manner [12]. This type of deployment requires for a high concentration of identical relay nodes to be employed in the communication environment and for information to be represented by a high number of excitons using sufficiently long optical pulses. The generated excitons for one bit of information diffuse through the channel, and if any of them reaches to the receiver, it is decoded into the original information. Communication range depends on the FRET characteristics, as well as the mobility of the relay fluorophores and their concentration in the communication channel. As the excited-state lifetimes and the mobility of the deployed relays are increased, the communication range is also expected to increase [12].

2) Switching: Control over the communication pathways on a nanonetwork is crucial for diversifying the applications. Although pathways can be predefined by exploiting the strong distance dependence of FRET [26], it is also possible to dynamically regulate the information flow on the nanonetwork through external control signals which can modulate one of the characteristics of the employed relay nodes, or act on the network topology by altering the geometry of nanostructures on which the communicating nodes are deployed. Switching of communication pathways can be realized by optical, electrical, chemical or biological means which are explained in detail as follows:

- Optical switching makes use of the photochromic molecules which are photoactive fluorophores that undergo reversible transformation between two states of different absorption spectral characteristics depending on the wavelength of irradiation [1]. While irradiation at visible wavelengths $(400 \mathrm{~nm}-700 \mathrm{~nm})$ switches their absorption at their characteristic spectrum off, ultraviolet (UV) irradiation switches their absorption back on. In this way, the extent of optical absorption is able to be reversibly modulated by an additional control signal. Since they do not change their states until the next stimulation, they 
(a)

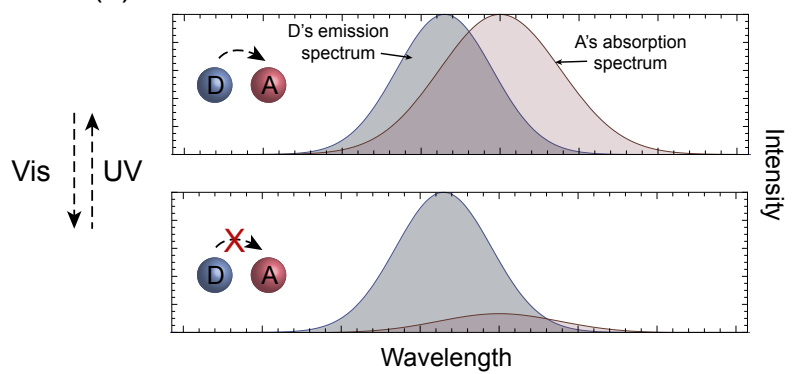

(c)

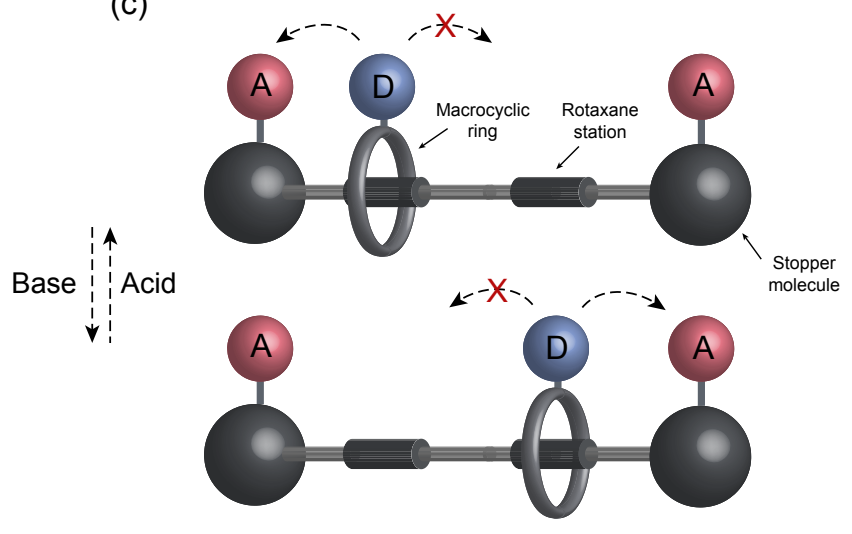

(b)

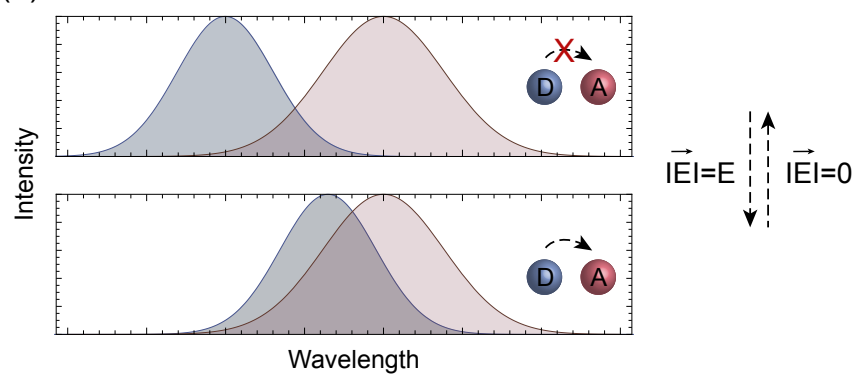

(d)

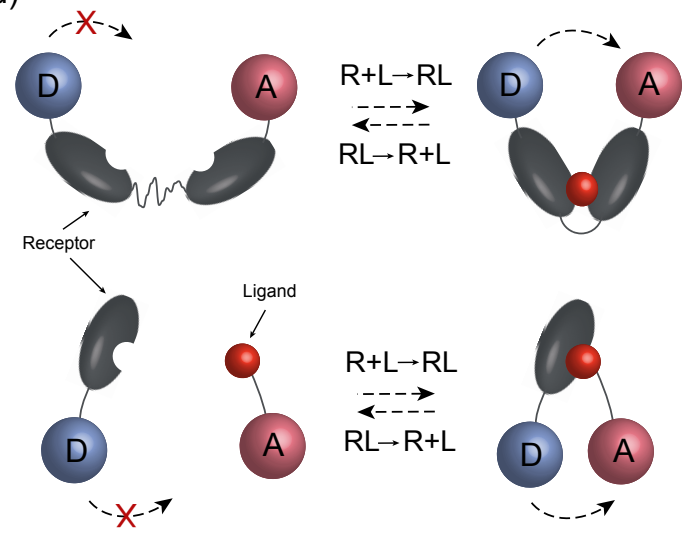

Fig. 3. Working principles of main switching mechanisms feasible for FRET-based nanonetworks: (a) Quenching of acceptor's absorption under visible light leading to optical switching; (b) Shift of donor's emission spectrum under electric field leading to electrical switching; (c) 1-d translational movement of macrocyclic ring of [2]rotaxane depending on the $\mathrm{pH}$ of environment leading to chemical switching; (d) Ligand-receptor binding leading to biological switching.

have been widely exploited as molecular memory units [5]. Photochromic fluorophores have also found use in FRET-based molecular switches [27], being employed as FRET acceptors adjacent to a spectrally similar donor fluorophore. Depending on the control signal, which is flipped between UV and visible wavelengths, the spectral overlap between the donor and photochromic acceptor is switched on and off leading to the modulation of FRET. This implies that a communication pathway relying on the flow of excitons could be optically controlled by employing photochromic molecules.

- Electrical switching is based on the Quantum Confined Stark Effect (QCSE) which defines the red-shift of the emission spectrum of a semiconductor fluorophore as proportional to the magnitude of the applied electric field [28]. Given the fact that QCSE is not observed in organic fluorophores, it can enable the modulation of the overlap between the emission spectrum of a semiconductor donor fluorophore and the absorption spectrum of an organic acceptor fluorophore with a varying electrical field supplied from an external source [29]. Modulation of spectral overlap leads to the control of communication pathways as the information flow from a semiconductor transmitter to an organic receiver fluorophore could be switched on and off depending on their spectral overlap controlled through electric field. If a semiconductor node is placed in between two organic nodes with proper spectral characteristics, information on the semiconductor node flows either toward the first node or toward the second node depending on the electric field [11].

- Chemical switching relies on the use of chemically active supramolecules, the physical forms of which change depending on a chemical input [30]. One of the examples is [2]rotaxane which comprises a macrocyclic ring trapped on the axis of a dumbbell-like component containing two recognition sites on top of it. Two stopper molecules are placed at the both ends of the dumbbell [31]. Depending on the $\mathrm{pH}$ of the environment, the ring on the axis undergoes a reversible translational movement between two recognition sites as a bistable molecular shuttle. When two fluorophores acting as acceptor are placed on the stopper molecules, and a donor flurophore is placed on the ring, due to the strong distance dependence of FRET, the flow of excitons from the donor to the acceptors is modulated with the $\mathrm{pH}$ level [32]. If this configuration is deployed in a nanonetwork, the information coming to the transmitter node on the ring flows either to the receiver node at the first recognition site or to the receiver at the second site. In this way, chemical switching of the communication pathways could be enabled [11]. Rotaxane-like switches can also be built using DNA [33], which could be better adapted into the FRET-based nanonetworks implemented on DNA chains.

- Biological switching can be realized based on the mod- 
ulation of intermolecular distance between donor and acceptor or the addition of another acceptor to the network through ligand-receptor binding [34]. It is the underlying mechanism of FRET-based biosensors which find extensive use in bioimaging applications. If a network node is placed on a receptor, and a strong acceptor fluorophore is bound to the corresponding ligand, binding of the ligand to the receptor brings the acceptor into the close proximity of the network node creating a new pathway for the excitons flowing through the network. Thus, the information flow in the initial pathway is switched on and off with the reversible binding of a ligand. Another strategy can be the placement of two network nodes on a receptor molecule, the structure of which changes upon binding of a ligand. This way, the nodes get closer to each other when a ligand binds to the receptor, allowing excitons to flow from one node to another. In this way, depending on the network topology, new communication pathways could be generated, or the existing pathways could be switched off with the presence of ligands on the communication environment.

3) Point-to-Multipoint Communication: A single transmitter node can communicate to multiple receiver nodes if the individual transmitter-receiver pairs satisfy the FRET criteria [11]. However, this is only possible when one of the multiexciton schemes is applied for the modulation, since a single exciton can only be transferred to a single receiver at a time and cannot be copied on the transmitter [16]. Representing the information with a finite length pulse, multiple excitons are generated on the transmitter and transferred to the receivers in a random manner. However, this does not guarantee that all of the receivers can get the information at the end of bit interval. If a receiver is closer to the transmitter than the other receivers, then, it has higher probability of receiving the information because of the stronger Förster coupling.

Broadcasting of information from the transmitter is also possible for the multihop communication case where identical relays are randomly distributed in a fluidic environment, and the information carrying excitons are diffusing through the lattice of relay nodes similar to the diffusion of molecules in molecular communication [12]. If there are multiple receivers with absorption spectra overlapping with the emission spectra of the relays, then they can receive the broadcasted information when they are in the diffusion range of excitons.

4) Multiple Access: As was discussed in the previous section, FRET is based on the pairwise coupling of fluorophores, and FRET-based communications does not include an actual propagation channel. Therefore, we cannot envision a multiple access scheme for single-hop FRET-based communications. This functionality on a nanonetwork can be realized if a multihop communication is performed [12]. Since the transferred excitons individually cannot carry an addressing information, multiple-access is possible only through timedivision multiplexing.

Other than the multiple access schemes, simultaneous communication of multiple transmitter-receiver pairs on the same medium can also be realized if different groups of relay nodes with divergent spectral properties are deployed with a high concentration [12]. In this way, excitons carrying the information of different transmitters hop through the different relays without interfering with each other, and independently reach to their target receivers. This is realized through wavelengthdivision multiplexing; however, the scheme is fundamentally different from the conventional wavelength-division multiple access, because the communicating pairs do not share the same physical channel, rather, they communicate through different multihop FRET links created on relay fluorophores with divergent spectral characteristics. The extent of co-channel interference in this deployment depends on the spectral similarity of the relay nodes employed on separate links.

5) Macro-Nano Interface: Fluorophores, being able to be optically excited and fluoresce, intrinsically behave as optical interfaces between macroscale and nanoscale worlds. Therefore, every node in a FRET-based nanonetwork actually acts as a macro-nano interface. In this sense, the characteristic absorption and emission spectra of an interface can be viewed as its natural addresses for sending and receiving optical messages. However, deploying fluorophores with different spectral addresses for every node is impractical for large-scale nanonetworks, considering that the spectrum, in which fluorophores can absorb and emit, is a limited source. Moreover, addresses cannot be defined by a single wavelength but a wavelength range, which decreases the maximum number of uniquely definable addresses in a nanonetwork. Hence, grouping large number of nodes based on their functionality in an application may be required [8].

Utilizing a tunable-wavelength laser as a gateway unidirectionally connecting a macroscale network to a nanonetwork, information can be sent to a specific group of nodes using the group's absorption address. On the other hand, information flow from a nanonetwork to a macroscale network can be realized by using a photodetector as a gateway [15]. Decoding of the information can be performed based on the wavelength of the detected fluorescence. In that way, bidirectional communication could be established between macroscale and nanoscale networks. Using a wavelength division multiplexing scheme in both link, the communication capacity of the macronano interface can be substantially improved.

For in vivo applications of FRET-based nanonetworks, tissue penetration depth of the light used for the excitation should also be considered. Two-photon excitation scheme developed for biological imaging applications enables the use of nearinfrared (NIR) light, which provides substantially improved tissue penetration compared to visible light exploiting the nearinfrared window in biological tissues [35]. The scheme is based on the excitation of a donor fluorophore through successive absorption of two photons with lower energy than the donor's normal absorption spectrum. In this way, a fluorophore normally absorbing at visible wavelengths can be excited by two NIR photons. Two-photon excitation could be also applied to FRET-based networks in living organisms by representing information on the external gateway with short pulses of NIR light in a single-exciton encoding scheme. 

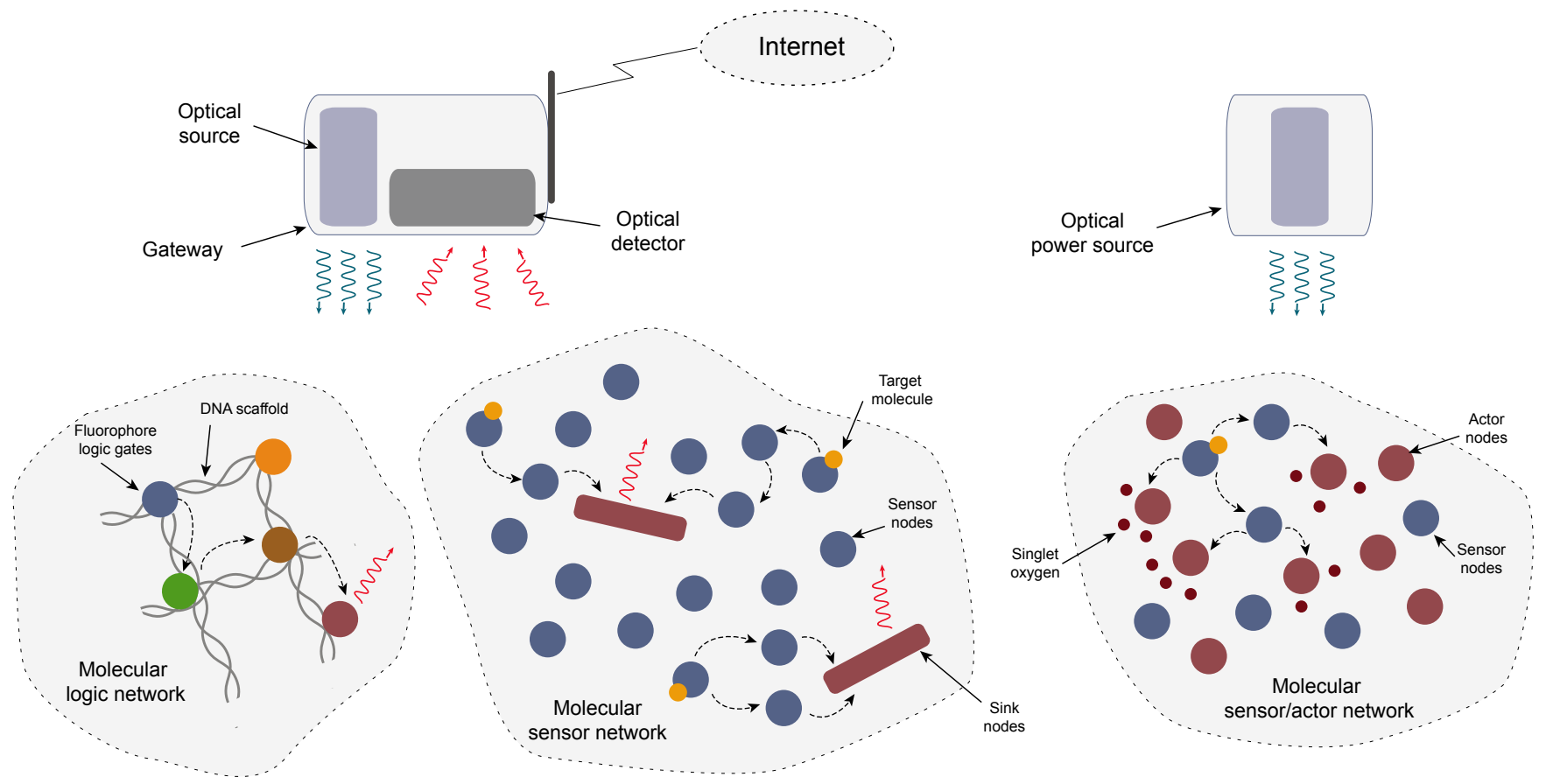

Fig. 4. Conceptual drawings of some applications of FRET-based communications. Molecular logic network and sensor network are connected to Internet via macroscale gateways comprising optical source and detectors whereas molecular sensor/actor network is envisioned to operate in autonomous manner under continuous excitation of a remote optical source.

\section{Applications}

FRET does not necessitate a specific environment, e.g., fluidic medium or free space, to be realizable; however, the efficiency of the energy transfer, thus the performance of the FRET-based communications, depends on the refractive index of the medium where the fluorophore network is deployed, as will be theoretically modeled in Section V. The network nodes can be mobile or immobile depending on the application and the medium.

In this section, we review some prospective applications and deployment scenarios of FRET-based nanocommunications. We investigate the applications under three main categories: i) molecular information processing; ii) collaborative sensing and actuating; and iii) molecular communications. Specifically, molecular information processing can be considered for immobile deployments, since the intermolecular distances between fluorophore-based logic nodes should be fixed to produce stable logical outputs. FRET-based information processing networks can be deployed on prescribed locations over organic scaffolds like DNA or inorganic scaffolds like zeolite L, and can be integrated into future lab-on-a-chip devices. They can be utilized for in vivo information processing, or for in vitro applications regarding to molecular computers. FRET-based collaborative sensing and actuating is considered particularly for mobile ad hoc deployments in fluidic medium towards medical and environmental applications, where sensing and actuating molecules search for and act upon the target location. For the application to molecular communications (MC), FRETbased fluorophore networks are considered to be deployed on MC transmitter and receiver nanomachines. The scenarios are detailed in the following subsections.

\section{A. Molecular Information Processing}

In recent years, a great deal of effort has been devoted to the development of molecular scale binary logic gates which can handle optical or chemical signals [36]-[38]. An extensive number of simple molecular switches which are able to generate binary 1 or 0 depending on the input signals have been already developed and proven feasible indicating that digital information processing with molecules even in living cells is not a far-fetched idea [39], [40]. Towards the long-standing goal of molecular computers, there is also an ongoing research aimed at development of efficient and reliable techniques to connect these switches with each other to enable more advanced processing. Current approaches in this context are mostly based on the use of chemical signals to carry the output information of one logic gate to the input of another [41], [42]. However, communication with chemical signals is a very slow process, and its reliability is also another challenge. FRET-based communication can be an alternative solution to this problem, if the switches are made of fluorophores. Examples of fluorophore-based switches handling several form of control inputs have been discussed in Section III-B, as they can also allow the routing of information flow at nanonetworks. Establishing a FRET-based communication link, an exciton on a fluorophore-based switch generated as a result of a logic operation can be carried to the input of another gate in a fast and reliable manner. If the switches are based on the operation of photochromic molecules controlled by optical signals [4], communication through FRET could enable 
rapid all-optical molecular information processing which is limited only by the photoisomerization speed of photochromic compounds. Integrated sensing and processing is also possible if biological or chemical switches are used as logic gates [43]. These logic networks can be implemented on a single supramolecular structure like DNA allowing a controllable spacing and orientation of molecules, which is important for the reliability of information transfer. Individual gates can also be attached to separate molecules such that the communication of logic gates is enabled when these molecules bind to each other, which can bring another dimension to the molecular information processing.

\section{B. Collaborative Sensing and Actuating}

Fluorophores are widely used in bioimaging applications as molecular beacons due to their intrinsic interface capability. Their use as molecular beacons is mainly based on switching of fluorescence due to the conformational changes on the conjugated molecule upon the binding of another molecule or a chemical reaction sensitizing the fluorophore as reviewed in Section III-B. Most of the sensing applications require for a high number of molecular sensors to be deployed in a large area. This complicates the decoding of sensing information at the macroscale because of the high number of fluorescence signals, the release times and propagation directions of which are random. Reducing the number of interface channels without comprising the range of sensing application can be possible, if individual sensing data which are intrinsically encoded into excitons are collected by central nodes, i.e., sink nodes, possessing more advanced communication capabilities. This could be enabled by FRET-based communication techniques using sensor nodes as relays in a multihop communication manner [12], as depicted in Fig. 4. The central nodes can transmit the collected information to the macroscale gateways in the form of amplified and directed fluorescence [44], [45]. They can also process the data with FRET-enabled on-chip logic networks before transmitting, for example, by multiplexing data collected from different kind of sensors [43].

If the employed central nodes are capable of acting on the local environment, then autonomous sensing and acting applications could be enabled. A particular application of FRET-based sensor/actor nanonetworks to the photodynamic therapy of cancer was investigated in [13], [14]. Photodynamic therapy is based on the use of photosensitizing agents that can be excited by a light source and sensitize singlet oxygens through the transfer of excited-state energy to an $\mathrm{O}_{2}$ molecule [6], [46], [47]. Singlet oxygen is extremely harmful for living cells. This property is exploited to initiate the apoptosis of cancer cells by producing them inside or in close proximity of these targets. However, as in the case of other cancer therapies, photodynamic therapy suffers from the low level selectivity, such that normal cells are also killed through singlet oxygens when they are not produced in target locations. A FRET-based ad hoc networking approach employing sensor nodes that can be excited upon the detection of target, and actor nodes which can synthesize singlet oxygens could substantially improve the targeting [14]. This could be enabled through multihop communication of these sensor nodes with the actor nodes. Since the excitons are mortal information packets, the information diffuses through a narrow spatial range in a very fast manner, hence, it is almost guaranteed for singlet oxygens to be produced by actor nodes in the close proximity of target cells.

\section{Application to Molecular Communications}

Fluorophores and FRET-based nanocommunications can also find use in molecular communications where molecules are used to encode, propagate, and decode information [48]. For example, fluorophore-based biological switches can be used as interface transferring the molecular information in an MC nanonetwork to the macroscale gateways in the form of fluorescence signals. If the specialized bacteria which can express fluorescent proteins [49] are employed as the communicating nanomachines, then the molecular information can be directly detected by macroscale gateways without requiring a bio-switching mechanism. The reverse case, where the molecular communication is optically controlled through macroscale gateways is also possible, if photoactive molecular compounds that can undergo isomerization based on the wavelength of optical signal are used [50], [51]. For example, an MC transmitter can be equipped with photoactive molecules that control the molecular release mechanism, such that they transmit an information to the channel upon receiving a control signal from a macroscale gateway. These functionalities can also be diversified with FRET-enabled fast processing capabilities of fluorophores on the transmitter and receiver nanomachines. If the received information molecules are transduced to excitons by the fluorophore bio-switches employed on the receiving unit, the information could be processed in the form of excitons, and then transmitted to the macroscale gateways, or used to release an action. Likewise, the transmitter could process the optical control signals together with the chemical inputs, and act, e.g., transmit a molecular message, according to the result of logic operation.

\section{Theoretical Modeling Approaches}

Communication and information theoretical modeling of FRET-based communications relies mainly on the FRET theory, which is established in Theodor Förster's seminal work [10] for immobile fluorophores, and then, validated by an extensive number of experimental studies [52]. For the cases where single-exciton encoding scheme is used, we are able to derive analytical expression for the capacity of the channel [9]. However, for multi-exciton encoding scheme [11], correlated exciton dynamics take part in the information transfer, which impedes the derivation of analytical models. Fortunately, communication performance metrics for multi-exciton cases can be revealed by conducting realistic Monte Carlo simulations. In this section, we first review the fundamentals of FRET theory, which will be then used for modeling the communication channel for single-exciton schemes. We also elaborate on the simulation algorithms, which were developed based on exciton dynamics to be adapted for various network configurations. 


\section{A. Theory of FRET}

As explained earlier, in the absence of any external quenching mechanisms, e.g., FRET, an excited-state donor returns to ground-state after a random short time by fluorescing. The excited-state lifetime, which is also the sojourn time of the corresponding exciton, $\tau$ can be expressed as an exponential random variable with mean $\mu_{0}$ [1]. The reciprocal of the mean excited-state lifetime equals to the natural fluorescence rate $k_{0}$ of a fluorophore, i.e., $k_{0}=1 / \mu_{0}$, which is the number of photons released at a unit time while the donor is continuously excited by a stimulation. Involvement of any quenching mechanism creates additional relaxation pathways for the excited-state donor, which reduces the sojourn time of excitons by increasing the overall relaxation rate of the donor. Considering the case when there are $k$ acceptors in close proximity of the excited donor, the reduced mean excited-state lifetime is given as follows

$$
\mu=\left(k_{0}+\sum_{j=1}^{k} k_{T, j}\right)^{-1}
$$

where $k_{T, j}$ is the FRET rate between the excited donor and the $j$ th acceptor surrounding the donor. $k_{T, j}$ in terms of the donor-acceptor spacing can be expressed by

$$
k_{T, j}=k_{0} \frac{R_{0, j}^{6}}{R_{j}^{6}} \mathbf{1}_{j}
$$

where $R_{0, j}$ is the Förster radius of the donor and the $j$ th acceptor, $R_{j}$ is the spacing between them, and $\mathbf{1}_{j}$ is an indicator function which indicates whether the $j$ th acceptor is in the ground-state or not, i.e., $\mathbf{1}_{j}=1$ if it is in the groundstate, otherwise $\mathbf{1}_{j}=0$. Note that when an acceptor is in the excited-state, it cannot accept further exciton until it relaxes [16]. Förster radius is the intermolecular distance at which the FRET rate is equal to the natural fluorescence rate of the donor [1], and incorporates the effect of numerous system parameters on the transfer rate:

$$
R_{0, j}^{6}=8.8 \times 10^{22} \kappa_{j, i}^{2} n^{-4} J_{j}(\lambda)
$$

where $\kappa_{j, i}^{2}$ is the orientation factor which gives a measure of relative orientation between the transition dipoles of donor and $j$ th acceptor; $n$ is the refractive index of the medium, and $J_{j}(\lambda)$ is the overlap measure between the emission spectrum of the donor and the absorption spectrum of the $j$ th acceptor. The orientation factor can be given as

$$
\kappa^{2}=\left(\cos \theta_{T}-3 \cos \theta_{D} \cos \theta_{A}\right)^{2}
$$

where $\theta_{T}, \theta_{D}$ and $\theta_{A}$ are the angles related to the geometry of transition dipoles which are demonstrated in Fig. 1. From this expression, we can infer that when the transition dipoles are orthogonal to each other, $\kappa^{2}$ will be 0 , and FRET does not occur. For the configurations where fluorophores are free to rotate in all there axes, or a FRET pair is self-assembled on a backbone structure without any control over transition dipoles, $\kappa^{2}$ would be a random variable with the following probability distribution [53]:

$$
p_{\kappa^{2}}\left(\kappa^{2}\right)= \begin{cases}\frac{1}{2 \sqrt{3 \kappa^{2}}} \ln (2+\sqrt{3}) & 0 \leq \kappa^{2} \leq 1 \\ \frac{1}{2 \sqrt{3 \kappa^{2}}} \ln \left(\frac{2+\sqrt{3}}{\sqrt{\kappa^{2}}+\sqrt{\kappa^{2}-1}}\right) & 1 \leq \kappa^{2} \leq 4\end{cases}
$$

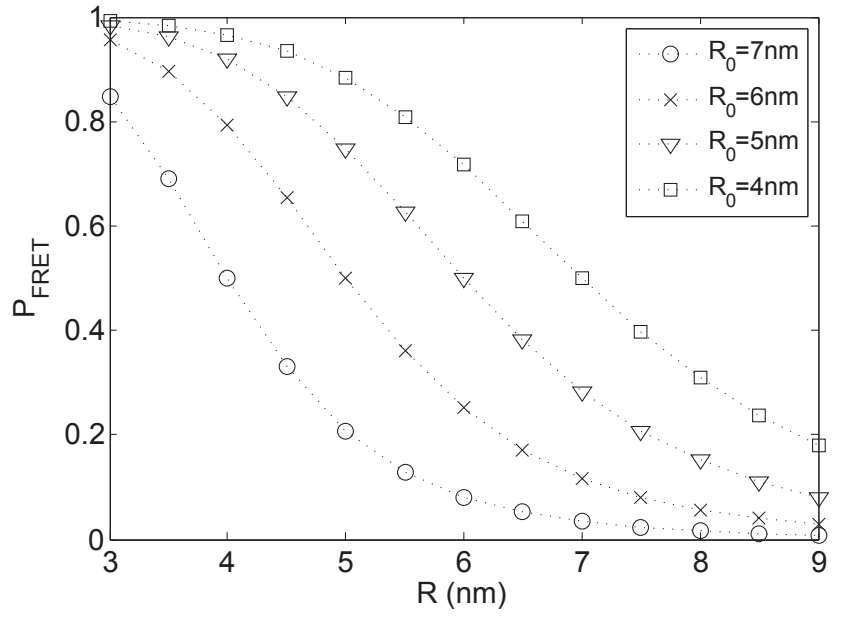

Fig. 5. Probability of FRET for an excited donor with varying donor-acceptor distance $R_{0}$ and Förster radius $R_{0}$.

In many experimental works studying an ensemble of FRET pairs, mean value of $\kappa^{2}$, i.e., $2 / 3$ is used with the assumption of free rotation [1].

Using the process rates, the probability of transfer for an excited donor can be given as

$$
P_{F R E T, j}=\frac{k_{T, j}}{k_{0}+\sum_{l=1}^{k} k_{T, l}}
$$

When there is only a single pair of donor and acceptor, this expression reduces to:

$$
P_{F R E T}=\frac{k_{T}}{k_{0}+k_{T}}
$$

Probability of FRET between a donor and acceptor fluorophore for different $R_{0}$ and $R$ is given in Fig. 5, which also reveals the strong distance dependence of FRET.

Now consider that a single exciton is transferred through cascaded FRET channels in a multihop manner on a one dimensional axis. The cascaded channels are shown to be independent of each other [24], [9]. Therefore, the probability of exciton transfer through $N$ cascaded FRET channels is given by

$$
P_{F R E T}=\prod_{i=1}^{N} P_{F R E T, i}
$$

where $P_{F R E T, i}$ is the transfer probability on the $i$ th channel.

In most cases, the assumption of immobility can also be applied to mobile fluorophores, because the process rates, i.e., fluorescence and FRET, are generally very much higher than the rate of translational movement, so that the fluorophores are almost immobile during an average lifetime of excited-state [1]. However, when the natural excited-state lifetimes or the diffusion coefficients of fluorophores are high enough that the excited-state molecules move distances comparable to Förster radius, then the mobility cannot be neglected. Averaging over all possible intermolecular distances, a rate expression is derived in [54] for mobile fluorophores as follows

$$
k_{T, r d}=\frac{4 \pi k_{0} R_{0}^{6}}{3 V a^{-3}}
$$




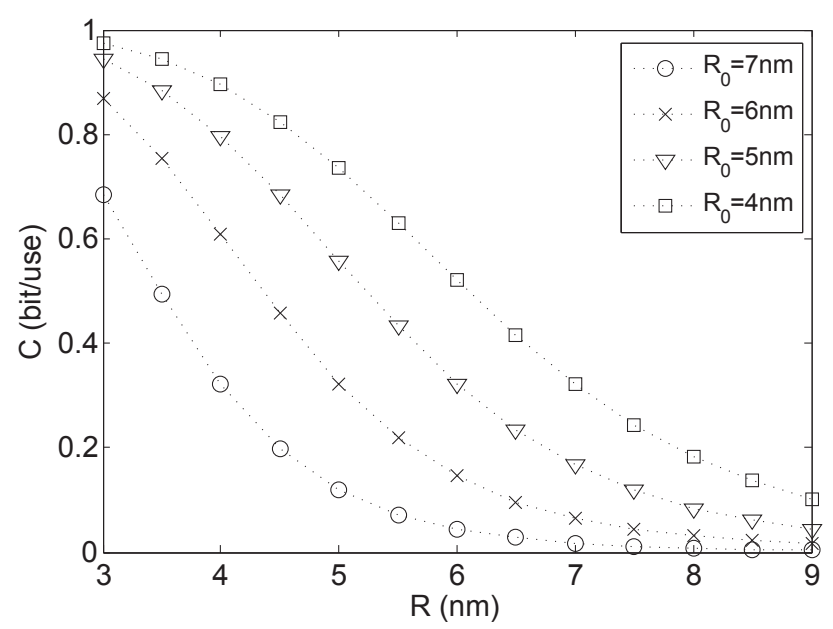

Fig. 6. Capacity of a point-to-point channel for different Förster radii $R_{0}$ and varying internodal distance $R$.

where $R_{0}$ is the Förster radius when $\kappa^{2}=2 / 3, V$ is the volume of the medium, $a$ is the distance of closest approach of donor and acceptor as measured between their centers. This expression is valid only when the fluorophores meet the rapiddiffusion criterion which is given by

$$
\frac{D \mu_{0}}{s^{2}} \gg 1
$$

where $D$ is the sum of the diffusion coefficients of the donor and acceptor, and $s$ is the mean intermolecular distance [54], [55]. Lanthanide complexes with lifetimes in the $\mu \mathrm{s}-\mathrm{ms}$ range can meet the criterion with moderate diffusion coefficients [56].

\section{B. Communication with Single-Exciton Encoding}

Consider the case when the information is encoded into single exciton with on-off keying modulation such that bit1 and bit- 0 are represented by the excited-state and groundstate of the fluorophores during a predefined bit interval $T_{b}$. If there is a single excitation source, bit- 0 can be sent to the receiver node without an error assuming the bit interval is large enough to avoid intersymbol interference (ISI). On the other hand, the reliability of bit-1 transmission strongly depends on the dynamics of exciton transfer. Successful transmission probability of bit-1 can be given as equal to the FRET probability [9];

$$
p_{1}=P_{F R E T}
$$

This expression is valid for all configurations, e.g., pointto-point, multihop and mobile communications, though the probability of FRET is given in terms of different parameters for different configurations.

During an information transfer, when bit- 0 is preceded by bit-1, ISI may occur if the bit interval is not set large enough that the receiver may still remain in excited-state at the time of bit- 0 transmission. Thus, the minimum $T_{b}$ that guarantees noISI condition depends on the excited-state lifetimes of both the transmitter and receiver. It should be greater than the maximum possible sojourn time of an exciton on the system;

$$
T_{b-\min }=\tau_{T, \max }+\tau_{R, \max }
$$

where $\tau_{T, \max }$ and $\tau_{R, \max }$ are the maximum of reduced and natural excited-state lifetimes of transmitter and receiver, respectively. Since the lifetimes are exponential random variables, $T_{b-\min }$ goes to infinity. Thus, we can never guarantee no-ISI condition. However, given the lifetimes are independently distributed, setting $T_{b}=4 \times\left(\mu_{\tau_{D A}}+\mu_{\tau_{A}}\right)$ results in ISI probability lower than $10^{-3}$, which might be acceptable depending on the application.

The transition probabilities of bit- 1 and bit-0, when noISI condition can be assumed, indicate that the channel can be information theoretically modeled as a Z-channel [57]. Denoting the binary input and output alphabets as $\mathbf{X}$ and $\mathbf{Y}$, respectively, the mutual information can be expressed by:

$$
I(\mathbf{X} ; \mathbf{Y})=H\left(P[1] p_{1}\right)-P[1] H\left(1-p_{1}\right)
$$

where $H($.$) is the binary entropy function, and P[1]$ is the probability of encoding bit-1 on the transmitter. Then the capacity of the channel will be the supremum of $I(\mathbf{X} ; \mathbf{Y})$ over all input distributions, i.e., $C=\sup _{P_{1}} I(\mathbf{X} ; \mathbf{Y})$. As can be seen in Fig. 6, the channel capacity strongly depends on the distance between the communicating nodes, and reduces to unacceptable levels when the distance is greater than the Förster radius.

\section{Communication with Multi-Exciton Encoding}

When the information is encoded into single exciton, the reliability of the channel substantially decreases when the spacing between communicating nodes exceeds the Förster radius [9]. Reliability can be improved with multi-exciton schemes using longer excitation pulses to represent information [11]. However, channel modeling for multi-exciton schemes is a highly complex task even for single-hop communications considering that multiple excitons exist on the system at the same time which results in highly random and correlated transfer dynamics. If there are multiple mobile nodes for multihop communications, the problem becomes more complex as the correlation expands over the spatial domain [12]. For such cases, deriving analytical expressions for performance metrics is almost impractical. However, communication can be simulated to evaluate main performance metrics such as channel capacity, communication rate and delay.

In our previous studies, we developed realistic Monte Carlo algorithms that fully capture the dynamics of excitons during a bit-1 transmission for numerous channel and network configurations such as multihop communications in 1-d and 3-d channels [12], point-to-multipoint communications [11], mobile ad hoc sensor/actor networks [14]. The sample pseudocode given in Algorithm 1 combines all of the previous approaches into a simple one which is written for the transmission of bit-1 in a multihop configuration with mobile relay nodes randomly deployed in 3-d environment containing a single pair of transmitter and receiver nodes. Bit-1 is encoded in the transmitter by a $T_{p}$-length excitation pulse. If $T_{p}$ is set to a value much lower than the excited-state lifetime, then 


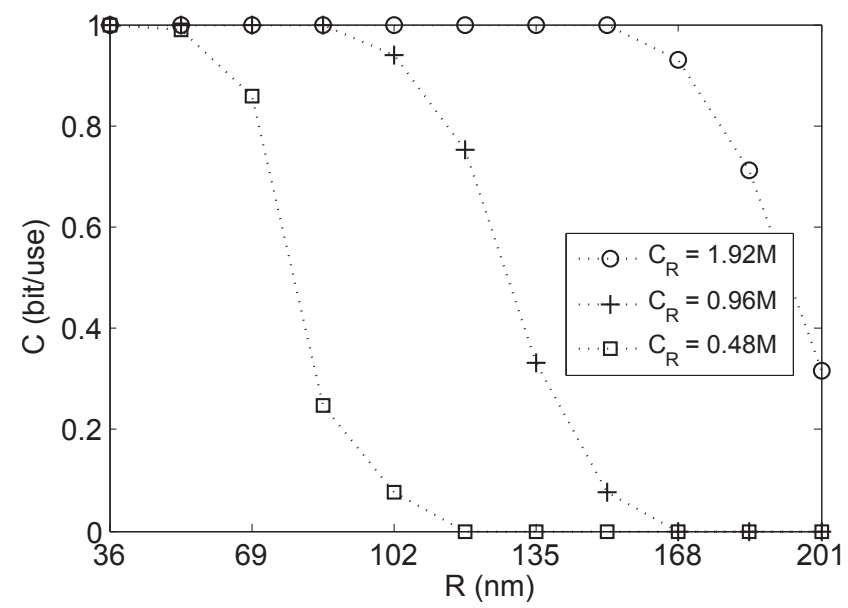

Fig. 7. Capacity of a multihop communication channel for different relay concentrations $C_{R}$ and varying transmitter-receiver distance. Data were obtained through Monte Carlo simulations using Algorithm 1 for $T_{p u l s e}=1 \mu \mathrm{s}$ and $R_{0}=6 \mathrm{~nm}$.

the encoding becomes single-exciton-based. The algorithm can be easily extended for MIMO configurations by setting the number of transmitter and receiver nodes to a value greater than one. The algorithm basically runs a single node at each loop based on their electronic state, and updates their state one by one for each time interval, which is set to a value small enough to guarantee that at most single event, e.g., fluoresce or FRET, can occur at a single time step. The nodes are assumed to undergo Brownian motion independently of the exciton dynamics at each time step. Simulations should be run until the observed parameter converges to a finite value. Although the algorithm is highly realistic and accurate, it becomes impractical and time-consuming for systems comprising of high number of nodes.

A simplified version of the algorithm is developed for the cases where the nodes meet rapid-diffusion criterion [13]. The pseudocode given in Algorithm 2 simulates the bit-1 transmission in a multihop communication between a pair of transmitter and receiver using mobile relays. It is based on a continuous time Markov process model which is given in Fig. 8. The numbered states of the process represent the number of excitons on the system, and state-D is the detection state which denotes the reception of information by the receiver. The model comprises of two processes; the first process model is valid during the application of $T_{p}$-length excitation pulse which encodes the information on the transmitter. In this process, both the generation and the recombination of excitons, i.e., removal by fluorescence, are possible together with the transfer among nodes, therefore, it denotes a birthdeath process. While the excitation continues, the transmitter generates excitons at a varying rate which depends on the electronic states of other network nodes. The upper limit for the state in the first process is the total number of nodes. When the excitation terminates, the system shifts into the second process model which represents a pure-death process with the initial state $N_{p}$ denoting the number of excitons on the system at time $T_{p}$. In the absence of the excitation

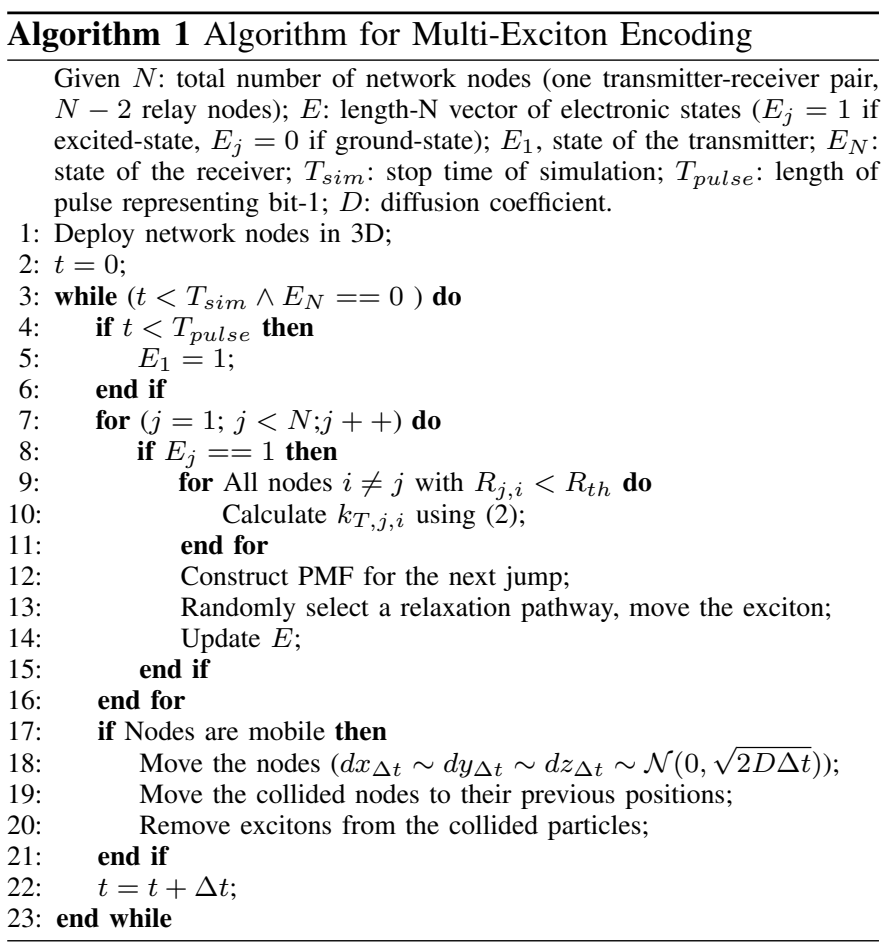

pulse, only the recombination and transfer of the previously generated excitons are possible. The simulation terminates when the receiver node detects an exciton, which is possible in both processes. The state-dependent transition rates for the processes are given by

$$
\begin{gathered}
\alpha_{i}=i k_{0} \\
\theta_{i}=i\left(N_{h}-i\right) k_{T} \\
\mu_{i}=i k_{T} \\
\beta_{i}=k_{0}+k_{T}\left(N_{h}-i\right)
\end{gathered}
$$

where $i$ is number of excitons on the network, $k_{T}$ denotes the transfer rates between nodes calculated using (9). Assuming that the time is divided into sufficiently small intervals $\Delta t$, the transition probabilities at each time step are calculated by multiplying the corresponding transition rates by $\Delta t$.

Markov process modeling approach simplifies the algorithm by averaging out the mobility and fixing the transfer rates, which should be determined at each time step in the previous algorithm. Although it is preferable for calculating the parameters that can be derived from transmission probabilities and delay, it is not possible using this algorithm to obtain spatial performance metrics such as communication coverage [13].

\section{EXPERIMENTAL APPROACHES AND IMPLEMENTATION CHALLENGES}

FRET is a well-known phenomenon which is extensively experimented for its widespread use in bioimaging applications [58]. It has an already validated theory for both ensemble (ensembleFRET) and single molecular cases (smFRET) [59]. From the aspect of communications, well-documented experimental methodologies together with the intrinsic interface capability of fluorophore nodes provide an easier pathway for 


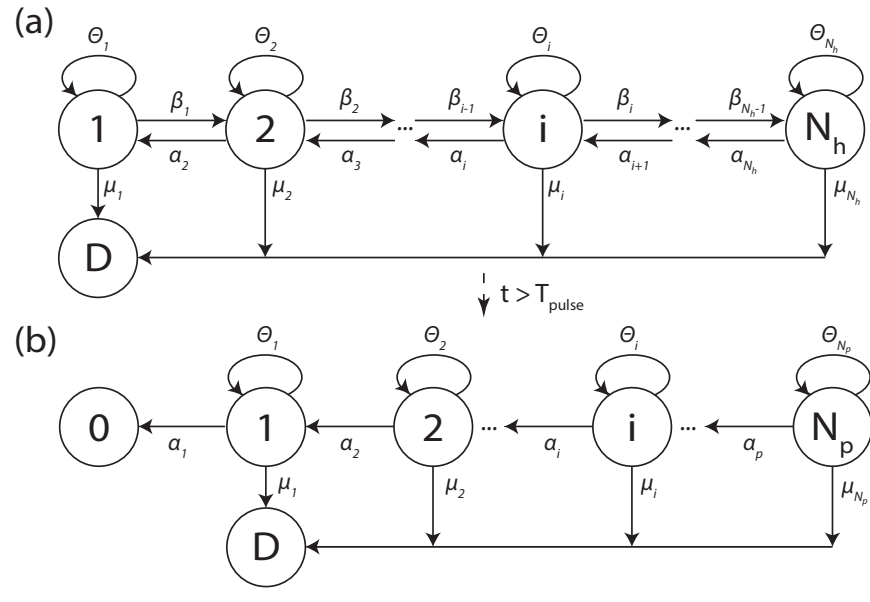

Fig. 8. Markov process model of bit-1 transmission for rapid-diffusion case: (a) Birth-death Markov process model valid for $t \leq T_{\text {pulse }}$; (b) Pure-death Markov process model valid for $t>T_{\text {pulse. }}$.

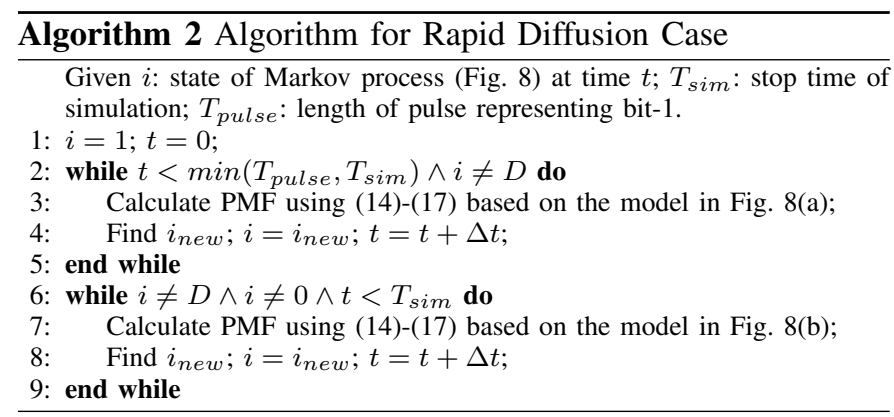

the validation of the theoretical models compared to other nanocommunications approaches. However, the experimental opportunities for single molecular cases, on which the most of the envisioned applications of FRET-based communication are based, are limited by the low sampling frequencies of stateof-the-art laboratory instruments, which are on the order of 1$10 \mathrm{kHz}$ for fluorescence measurements [60]. This would restrict the empirical studies of FRET-based communications, at best, to the rates below 10kbps. On the transmitter side, however, it is possible to encode the information even into single excitons using femtosecond-length lasers [61].

On the other hand, using an ensemble of molecular channels increases the intensity of output fluorescence; therefore, ensemble measurements are not subject to the strict limitations of smFRET [62]. In this way, recently, we have demonstrated the first real-time and high-rate information transmission between organic dyes, i.e., Fluorescein and Rhodamine B, in a FRETbased MIMO configuration, proving the feasibility of FRETbased nanoscale communications [15]. We transmitted 5Mbit pseudorandom binary data at 50, 150 and $250 \mathrm{kbps}$ rates with acceptable BER values, i.e., $1.91 \times 10^{-6}, 5.72 \times 10^{-5}$, $3.05 \times 10^{-2}$, respectively. We used a simple experimental setup including a Ar-Ion laser, the output of which is modulated based on OOK scheme by an electro-optical modulator. The transferred information is measured optically through a conventional photodetector after passing appropriate optical filters. One challenge associated with the observation of the received information is the spectral bleed-through (SBT) resultant from the contamination of the observation channel by the direct fluorescence of receiver molecules [64]. This is due to the fact that the receivers can be excited directly by the excitation pulse of laser if their absorption spectra overlap the excitation wavelength. Another reason of contamination is the direct fluorescence of the transmitters with wavelengths at the spectral range of the observation channel. These unintended contributions result in the contamination of the observed signal which should only contain the fluorescence of receivers due to FRET. Therefore, in most cases, post signal processing is required to obtained the purified FRET signal. 3-cube method is the most prevalent technique to purify the observed signal, which is based on processing of measurements obtained from three samples containing only donor, only acceptor, and the mix of donor and acceptor [63], [64].

Another challenge associated with implementing a FRETbased nanonetwork may be the photostability of fluorophores. Most fluorophores, especially organic dyes and fluorescent proteins, undergo photobleaching, i.e., lose their fluorescence and FRET capabilities, after a number of excitation-relaxation cycles, e.g., $10^{5}-10^{6}$ for organic dyes [1]. Reducing oxygen concentration in the environment increases the resistance of fluorophores to photobleaching [60]; however, it may not always be possible to remove oxygen from the communication medium, especially considering in vivo applications. Alternatively, the problem can be overcome by using intrinsically stable fluorophores such as semiconductor QDs and Nitrogenvacancy (NV) centers [65], [66], which have almost infinite resistance to photobleaching. Another way could be the use of single-exciton encoding schemes, which increases the service time of organic dyes because of the reduced intensity of irradiation.

Additionally, fluorescent proteins and semiconductor fluorophores under strong illumination could show random blinking whose origin has not been fully understood yet [60]. When they are used on the transmitter side, the node could lose its ability to generate exciton for short random intervals due to photoinduced blinking, which may hinder reliable communication. Blinking can be chemically suppressed with the addition of auxiliary compounds to the communication environment [67]. Using single-exciton scheme, in which the transmitter nodes are exposed to low-intensity illumination, can also reduce the probability of blinking [60].

For in vivo applications, another challenge is to optically connect the nanonodes with macroscale gateways because of the limited penetration length of visible light in tissue. As discussed in Section III-B5, this problem can be overcome by using two-photon excitation scheme which enables the use of NIR light, that allows much more tissue penetration exploiting the near-infrared window in biological tissues, for the downlink transmission [35]. To decrease the attenuation using NIR light for transmission in the uplink from nanonetwork to macroscale gateways, the transmitting nodes can be selected from near-infrared emitting fluorophores [68]. 


\section{CONCLUSION}

Developing networks of molecular-scale devices possessing very limited operational capabilities necessitates radically different communication strategies than the prominent nanonetworking approaches. In this paper, we have reviewed the theoretical and experimental positions toward realizing the internet of fluorophore-based molecular devices based on FRET. We have presented an overview of the basic principles and design requirements of FRET-based nanocommunications, its networking opportunities and prospective applications including molecular-scale information processing and cooperative sensing/actuating for disease treatment. Main implementation challenges, e.g., low photostability of fluorophores and sampling rate limitations of single molecular experiments, along with the possible solution pathways have been discussed. Theoretical modeling approaches, simulation algorithms and experimental opportunities together with networking strategies provided in this paper will pave the way for the development of unprecedented applications making use of high-rate, reliable and controllable information transfer between molecular devices.

\section{REFERENCES}

[1] J. R. Lakowicz, Principles of Fluorescence Spectroscopy, 3rd ed., Baltimore, MD: Springer-Verlag, 2006.

[2] S. W. Hell, "Microscopy and its focal switch," Nature Methods, vol. 6, no. 1, pp. 24-32, 2009.

[3] A. P. Silva, H. Q. N. Gunaratne, T. Gunnlaugsson, A. J. M. Huxley, Colin P. McCoy, J. T. Rademacher and T. E. Rice, "Signaling recognition events with fluorescent sensors and switches," Chem. Rev., vol. 97, no. 5, pp. 1515-1566, 1997

[4] J. Cusido, E. Deniz and F. M. Raymo, "Fluorescent switches based on photochromic compounds," European Journal of Organic Chemistry, vol. 2009, no. 13, pp. 2031-2045, 2009.

[5] S. Kawata and Y. Kawata, "Three-dimensional optical data storage using photochromic materials," Chem. Rev., vol. 100, no. 5, pp. 1777-1788, 2000.

[6] A. C. S. Samia, X. Chen and C. Burda, "Semiconductor quantum dots for photodynamic therapy," J. Am. Chem. Soc., vol. 125, no. 51, pp. 1573615737, 2003.

[7] I. F. Akyildiz and J. M. Jornet, "The internet of nano-things," IEEE Wireless Communications, vol. 17, no. 6, pp. 58-63, 2010.

[8] S. Balasubramaniam and J. Kangasharju, "Realizing the internet of nano things: Challenges, solutions, and applications," Computer, vol. 46, no. 2, pp. 62-68, 2013.

[9] M. Kuscu and O. B. Akan, "A physical channel model and analysis for nanoscale molecular communications with Förster resonance energy transfer (FRET)," IEEE Trans. Nanotechnol., vol. 11, pp. 200-207, 2012.

[10] T. Förster, "Zwischenmolekulare energiewanderung und fluoreszenz", Annalen der Physik, vol. 437, pp. 55 - 75, 1948.

[11] M. Kuscu and O. B. Akan, "FRET-based nanoscale point-to-point and broadcast communications with multi-exciton transmission and channel routing," IEEE Trans. Nanobiosci., vol. 13, no. 3, pp. 315-326, 2014.

[12] M. Kuscu and O. B. Akan, "Multi-step FRET-based long-range nanoscale communication channel," IEEE J. Sel. Areas Commun., vol. 31, no.12, pp. 715-725, 2013.

[13] M. Kuscu and O. B. Akan, "A communication theoretical analysis of FRET-based mobile ad hoc molecular nanonetworks," IEEE Trans. Nanobiosci., vol. 13, no. 3, pp. 255-266, 2014.

[14] M. Kuscu and O. B. Akan, "Coverage and throughput analysis for FRETbased mobile molecular sensor/actor nanonetworks," Nano Commun. Netw., vol. 5, no. 1-2, pp. 45-53, 2014.

[15] M. Kuscu, A. Kiraz and O. B. Akan, "Fluorescent molecules as transceiver nanoantennas: The first practical and high-rate information transfer over a nanoscale communication channel based on FRET," Sci. Rep., vol. 5, pp. 7831, 2015.

[16] C. Berney and G. Danuser, "FRET or no FRET: A quantitative comparison," Biophysics Journal, vol. 84, no. 6, pp. 3992-4010, 2003.
[17] L. P. Silva and J. C. G. E. Silva, "Firefly chemiluminescence and bioluminescence: Efficient generation of excited states," ChemPhysChem, vol. 13, no. 9, pp. 22572262, 2012.

[18] J. Proakis and M. Salehi, Digital Communications, 5th ed., New York: McGraw-Hill, 2008.

[19] J. J. Toppari, J. Wirth, F. Garwe, O. Stranik, A. Csaki, J. Bergmann, W. Paa and W. Fritzsche, "Plasmonic coupling and long-range transfer of an excitation along a DNA nanowire," ACS Nano, vol. 7, no. 2, pp. 1291-1298, 2013.

[20] K. A. Velizhanin and T. V. Shahbazyan, "Long-range plasmon-assisted energy transfer over doped graphene," Phys. Rev. B, vol. 86, no. 245432, 2012.

[21] M. Kuscu and O. B. Akan, "An information-theoretic model and analysis of graphene plasmon-assisted FRET-based nanocommunication channel," in Proc. ACM NANOCOM, Atlanta, GA, USA, 2014.

[22] G. Bulgarini, M. E. Reimer, M. Hocevar, E. P. A. M. Bakkers, L. P. Kouwenhoven and Val Zwiller, "Avalanche amplification of a single exciton in a semiconductor nanowire," Nature Photonics, vol. 6, no. 7, pp. 455-458, 2012.

[23] I. F. Akyildiz, J. M. Jornet and M. Pierobon, "Nanonetworks: A new frontier in communications," Commun. ACM, vol. 54, pp. 84-89, 2011.

[24] M. Heilemann, P. Tinnefeld, G. S. Mosteiro, M. G. Parajo, N. F. Hulst and M. Sauer, "Multistep energy transfer in single molecular photonic wires," J. Am. Chem. Soc., vol. 126, no. 21, pp. 6514-6515, 2004.

[25] S. Ramachandra, Z. D. Popovic, K. C. Schuermann, F. Cucinotta, G. Calzaferri and L. De Cola, "Förster resonance energy transfer in quantum dotdye-loaded zeolite L nanoassemblies", Small, vol. 7, no. 10, pp. 14881494, 2011.

[26] I. H. Stein, C. Steinhauer and Philip Tinnefeld, "Single-molecule fourcolor FRET visualizes energy-transfer paths on DNA origami", J. Am. Chem. Soc., vol. 133, no. 12, pp. 41934195, 2011.

[27] F. M. Raymo and M. Tomasulo, "Electron and energy transfer modulation with photochromic switches", Chem. Soc. Rev., vol. 34, no. 4, pp. 327-336, 2005.

[28] S. A. Empedocles and M. G. Bawendi, "Quantum-confined stark effect in single CdSe nanocrystallite quantum dots," Science, vol. 278, pp. 2114 2117, 1997.

[29] K. Becker, J. M. Lupton, J. Müller, A. L. Rogach, D. V. Talapin, H. Weller and J. Feldmann, "Electrical control of Förster energy transfer," Nature Materials, vol. 5, pp. 777-781, 2006.

[30] P. R. Ashton, R. Ballardini, V. Balzani, I. Baxter, A. Credi, M. C. T. Fyfe, M. T. Gandolfi, M. Gomez-Lopez, M. Martinez-Diaz, A. Piersanti, N. Spencer, J. F. Stoddart, M. Venturi, A. J. P. White and D. J. Williams, "Acid-base controllable molecular shuttles," J. Am. Chem. Soc., vol. 120, no. 46, pp. 11932-11942, 1998.

[31] A. R. Pease, J. O. Jeppesen, J. F. Stoddart, Y. Luo, C. P. Collier and J. R. Heath, "Switching devices based on interlocked molecules," Acc. Chem. Res., vol. 34, no. 6, pp. 433-444, 2001.

[32] Y. Li, H. Li, Y. Li, H. Liu, S. Wang, X. He, N. Wang and D. Zhu, "Energy transfer switching in a bistable molecular machine," Organic Letters, vol. 7, no. 22, pp. 4835-4838, 2005.

[33] D. Ackermann, T. L. Schmidt, J. S. Hannam, C. S. Purohit, A. Heckel and Michael Famulok, "A double-stranded DNA rotaxane," Nature Nanotechnol., vol. 5, no. 6, pp. 436-442, 2010.

[34] A. Ibraheem and R. E. Campbell, "Designs and applications of fluorescent protein-based biosensors," Curr. Opin. Chem. Biol., vol. 14, no. 1, pp. 30-36, 2010 .

[35] M. Pawlicki, H. A. Collins, R. G. Denning and H. L. Anderson, "Twophoton absorption and the design of two-photon dyes," Angew. Chem. Int. Edit, vol. 48, no. 18, pp. 3244-3266, 2009.

[36] F. M. Raymo and S. Giordani, "Signal processing at the molecular level," J. Am. Chem. Soc., vol. 123, no. 19, pp. 4561-4562, 2001.

[37] K. Szacilowski, "Digital information processing in molecular systems," Chem. Rev., vol. 108, no. 9, pp. 34813548, 2008.

[38] U. Pischel, J. Andreasson, D. Gust and V. F. Pais, "Information processing with molecules - Quo vadis?," ChemPhysChem, vol. 14, no. 1, pp. 28-46, 2013.

[39] A. Credi, "Molecules that make decisions," Angew. Chem. Int. Ed., vol. 46, no. 29, pp. 5472-5475, 2007.

[40] A. P. Silva and S. Uchiyama, "Molecular logic and computing," Nature Nanotechnol., vol. 2, pp. 399-410, 2007.

[41] S. Erbas-Cakmak and E. U. Akkaya, "Cascading of molecular logic gates for advanced functions: A self-reporting, activatable photosensitizer," Angew. Chem. Int. Ed., vol. 52, no. 43, pp. 11364-8, 2013.

[42] E. T. Ecik, A. Atilgan, R. Guliyev, T. B. Uyar, A. Gumusa and E. U. Akkaya, "Modular logic gates: cascading independent logic gates via metal ion signals," Dalton Trans., vol. 43, no. 1, pp. 67-70, 2014. 
[43] C. LaBoda, H. Duschl and C. L. Dwyer, "DNA-enabled integrated molecular systems for computation and sensing," Acc. Chem. Res., vol. 47, no. 6, pp. $18161824,2014$.

[44] T. Ming, H. Chen, R. Jiang, Q. Li and J. Wang, "Plasmon-controlled fluorescence: Beyond the intensity enhancement," J. Phys. Chem. Lett., vol. 3, pp. 191-202, 2012.

[45] G. Cosa, "Single-molecule fluorescence: Assembling nanoantennas," Nature Chemistry, vol. 5, pp. 159160, 2013.

[46] D. E. J. G. J. Dolmans, D. Fukumura and R. K. Jain, "Photodynamic therapy for cancer," JNature Reviews Cancer, vol. 3, pp. 380-387, 2003.

[47] W. R. Dichtel, J. M. Serin, C. Edder, J. M. J. Frechet, M. Matuszewski, L. Tan, T. Y. Ohulchanskyy and P. N. Prasad, "Singlet oxygen generation via two-photon excited FRET," J. Am. Chem. Soc., vol. 126, no. 17, pp. 53805381, 2004.

[48] T. Nakano, M. J. Moore, F. Wei, A. V. Vasilakos and J. Shuai, "Molecular communication and networking: opportunities and challenges," IEEE Trans. on Nanobiosci., vol. 11, no. 2, pp. 135-148, 2012.

[49] C. M. Southward and M. G. Surette, "The dynamic microbe: green fluorescent protein brings bacteria to light," NMolecular Microbiology, vol. 45, no. 5, pp. 1191-1196, 2002.

[50] N. K. Mal, M. Fujiwara and Y. Tanaka, "Photocontrolled reversible release of guest molecules from coumarin-modified mesoporous silica," Nature, vol. 421, pp. 350-353, 2003.

[51] W. R. Browne and B. L. Feringa, "Making molecular machines work," Nature Nanotechnol., vol. 1, pp. 25-35, 2006.

[52] L. Stryer, "Fluorescence energy transfer as a spectroscopic ruler," Апnи. Rev. Biochem., vol. 47, pp. 819-846, 1978.

[53] D. Badali and C. C. Gradinaru, "The effect of Brownian motion of fluorescent probes on measuring nanoscale distances by Förster resonance energy transfer", J Phys Chem., vol. 134, no. 22, pp. 225102, 2011.

[54] L. Stryer, "Diffusion-Enhanced Fluorescence Energy Transfer," Ann. Rev. Biophys. Bioeng., vol. 11, pp. 203-222, 1982.

[55] K. Kinosita, T. Kouyama and A. Ikegami, "Criteria for the rapid diffusion limit of fluorescence energy transfer," J. Chem. Phys., vol. 86, no. 6, pp. 3273-3282, 1987.

[56] J. Vuojola and T. Soukka, "Luminescent lanthanide reporters: new concepts for use in bioanalytical applications," Methods Appl. Fluoresc., vol. 2, no. 1, pp. 012001, 2014

[57] T. M. Cover and J. A. Thomas, Elements of information theory, New York: Wiley, 1991.

[58] E. A. Jares-Erijman and T. M. Jovin, "FRET imaging," Nature Biotechnol., vol. 21, pp. 1387-1395, 2003

[59] T. Ha, "Single-molecule fluorescence resonance energy transfer," Methods, vol. 25, no. 1, pp. 7886, 2001.

[60] R. Roy, S. Hohng, and T. Ha, "A practical guide to single-molecule FRET", Nature Methods, vol. 5, pp. 507-516, 2008

[61] S. Quentmeier, S. Denicke, J.-E. Ehlers, R. A. Niesner and K.-H. Gericke, "Two-color two-photon excitation using femtosecond laser pulses", J. Phys. Chem. B, vol. 112, no. 18, pp. 57685773, 2008.

[62] S. S. Vogel, C. Thaler and S. V. Koushik, "Fanciful FRET", Science STKE, vol. 2006, no. 331, pp. re2, 2006.

[63] M. Elangovana, H. Wallrabeb, Y. Chena, R. N. Dayc, M. Barrosob and A. Periasamya, "Characterization of one- and two-photon excitation fluorescence resonance energy transfer microscopy", Methods, vol. 29, no. 1 , pp. $5873,2003$.

[64] G. W. Gordon, G. Berry, X. H. Liang, B. Levine and B. Herman, "Quantitative fluorescence resonance energy transfer measurements using fluorescence microscopy", Biophys. J., vol. 74, no. 5, pp. 27022713, 1998.

[65] A. R. Clapp, I. L. Medintz and H. Mattoussi, "Förster resonance energy transfer investigations using quantum-dot fluorophores", ChemPhysChem, vol. 7 , no. 1 , pp. $4757,2006$.

[66] J. Tisler, R. Reuter, A. Lammle, F. Jelezko, G Balasubramanian, P. R. Hemmer, F. Reinhard, and J. Wrachtrup, "Highly efficient FRET from a single nitrogen-vacancy center in nanodiamonds to a single organic molecule", ACS Nano, vol. 5, no. 10, pp. 7893-7898, 2011.

[67] I. Rasnik, S. A. McKinney and T. Ha, "Nonblinking and long-lasting single-molecule fluorescence imaging," Nat. Methods, vol. 3, pp. 891-893, 2006.

[68] S. A Hilderbrand and Ralph Weissleder, "Near-infrared fluorescence: application to in vivo molecular imaging," Curr. Opin. Chem. Biol., vol. 14, pp. 71-79, 2009.

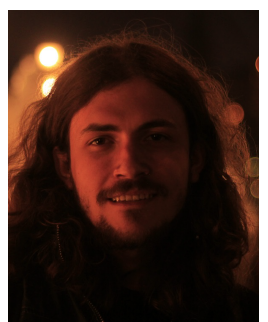

Murat Kuscu [S'11] (mkuscu@ku.edu.tr) received his B.Sc. degree in electrical and electronics engineering from Middle East Technical University, Ankara, Turkey, in July 2011, and his M.Sc. degree in Electrical and Electronics Engineering Department of Koc University, Istanbul, Turkey, in September 2013. He is currently a research assistant at the Next-generation and Wireless Communications Laboratory and pursuing his $\mathrm{Ph} . \mathrm{D}$. degree in electrical and electronics engineering at Koc University, Istanbul, Turkey. His current research interests include nanoscale and molecular communications.

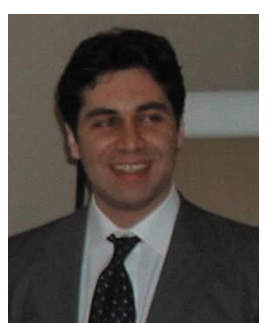

Ozgur B. Akan [M'00-SM'07] (akan@ku.edu.tr) received his $\mathrm{Ph} . \mathrm{D}$. degree in electrical and computer engineering from the Broadband and Wireless Networking Laboratory, School of Electrical and Computer Engineering, Georgia Institute of Technology in 2004. He is currently a full professor with the Department of Electrical and Electronics Engineering, Koc University, and the director of the Next-generation and Wireless Communications Laboratory. His current research interests are in wireless communications, nanoscale and molecular communications, and information theory. He is an Associate Editor of IEEE Transactions on Communications, IEEE Transactions on Vehicular Technology, IET Communications, International Journal of Communication Systems (Wiley), Nano Communication Networks Journal (Elsevier), and European Transactions on Technology. 